\title{
Decision support for selecting a shortlist of electricity-saving options: A modified SMAA approach
}

\author{
I Durbach* $\quad$ S Davis ${ }^{\dagger}$
}

Received: 15 December 2011; Revised: 7 August 2012; Accepted: 15 August 2012

\begin{abstract}
This paper describes an application providing decision support for generating a shortlist of promising electricity-saving options for households in South Africa. The decision problem is characterised by constraints on time and other resources, and by substantial uncertainty around the preferences for energy-related attributes and the performance of alternatives on those attributes. We use a stochastic multi-criteria acceptability analysis model to incorporate preferential uncertainties, and adapt this for use with quantiles and other "simplified" formats for representing uncertain attribute evaluations.
\end{abstract}

Key words: Multiple criteria decision analysis, decision support systems, uncertainty modelling, energy sector.

\section{Introduction}

The rising importance of electricity has been called "perhaps the twentieth century's most far-reaching, long-term energy trend whose course is still far from over" [33]. At the point of consumption, electricity is clean, silent, effortlessly accessible and adjustable, and flexible in its final use. Yet there is considerable evidence that the current level of global energy consumption - electricity included - is at a level that may not be able to sustain the integrity of the biosphere [34]. Increasing attention is being paid to strategies that aim to reduce energy consumption at various levels of society. In South Africa these strategies have been the source of considerable public interest because at times peak demand for electricity has outstripped capacity, leading to failures of supply systems and lengthy periods of enforced blackouts for industry and public society.

\footnotetext{
${ }^{*}$ Corresponding author: Department of Statistical Sciences, University of Cape Town, Rondebosch, 7701, South Africa, email: ian.durbach@uct.ac.za

${ }^{\dagger}$ Energy Research Centre, University of Cape Town, Private bag, Rondebosch, 7701 , South Africa.
} 
This paper describes a part of a project commissioned by the South African national electricity supplier Eskom ${ }^{1}$ as part of its demand-side management (DSM) program. The project's primary objective is to develop tools which empower Eskom's residential customers to make more informed electricity consumption choices, in particular choices on how to reduce their consumption. The tools aim to help energy users to (a) be aware of the electricity saving options available to them; (b) be able to evaluate the options available; (c) be actively involved in decision making around their own energy-related behaviour. The focus of this paper is on a small part of this ongoing project: the design of decision support for addressing aim (a). The intention is to create awareness (at least partially) through the development of an information campaign making households aware of a limited number of "best ways to save electricity", to be distributed through print, internet, and other channels. Limiting the number of presented options is considered essential in light of the enormous number of possible ways to reduce electricity — some initial screening is required to avoid overloading users with information. The project itself is undertaken by the Energy Research Center (ERC), a multi-disciplinary research unit at the University of Cape Town focusing on research into energy, poverty and development; energy efficiency; energy systems analysis and planning; and the environment and climate change. The current paper describes a pilot study constructing decision support which assists the ERC to perform this screening. The decision support provided has the following two features which make it of broader interest:

- Support is provided not directly to a 'decision maker' but to a group tasked with selecting a shortlist of alternatives on behalf of individuals belonging to a market or 'population' for which the group is responsible. Each of the individuals making up the market will then make their own unfacilitated choice from the shortlist. Very little is known about the preferences of the individuals making up the market, making a traditional value function approach impossible. We use stochastic multi-criteria acceptability analysis (SMAA), a family of inverse preference models based on an analysis of inverse weight spaces [3], to address this problem.

- Attribute evaluations are uncertain, and given time and resource constraints the use of probability distributions was not considered feasible. Current SMAA models treat uncertain attribute evaluations using probability distributions, thus requiring some adaptation to incorporate what we term "simplified" uncertainty formats quantities that in some way summarize the full probability distribution (e.g. expected values, variances, quantiles, etc.).

The remainder of the paper is structured as follows. Section 2 provides further detail on the project and describes the steps involved in structuring the decision problem. Section 3 describes the SMAA model used to provide decision support around the selection of the shortlist of options. Section 4 describes the results obtained from the simplified SMAA model, and Section 5 provides some reflections on the attempt to apply a simplified SMAA model to a practical decision problem.

\footnotetext{
${ }^{1}$ Eskom is a parastatal organisation responsible for the generation of approximately $95 \%$ of the electricity used in South Africa and $45 \%$ of the electricity used in Africa. It is regulated by the National Energy Regulator of South Africa under the Electricity Regulation Act and by the National Nuclear Regulator in terms of the National Nuclear Regulatory Act. See http://www.eskom.co.za/live/index.php.
} 


\section{Structuring the decision problem}

The following elements were considered while the decision model was developed.

\subsection{Research objectives}

This application follows the distinction drawn in the action-research literature between action aims and research aims [6]. Our primary action aim is to identify a shortlist of promising electricity savings options to be included on a media insert. It should be possible to customise this shortlist to certain types of users i.e. in the sense of a 'targeted' marketing campaign [24]. Following similar applications of simplified multi-criteria decision models (MCDA) models in [32], the research aims here are (a) to establish whether a simplified SMAA model could be employed in a practical decision problem; (b) to identify any difficulties experienced during the application, and (c) to establish whether the additional decision support provided by incorporating assessments of uncertainty was perceived by decision makers to be useful.

\subsection{Stakeholder involvement}

Ultimate responsibility for formulating electricity saving policy lies with Eskom. Their decisions are based on multiple sources of information collected from external sources predominantly previous literature and studies conducted by consulting groups such as the ERC. In this application the stakeholder and source of information is a group of energy researchers at the ERC who are primarily responsible for the project. This group provided model inputs and feedback on the modelling process and outputs. Electricity-consuming households are not directly represented in the decision process.

\subsection{Constructing attributes and alternatives}

The set of attributes and alternatives to be used was essentially given through the work of another group of researchers at Sustainable Energy Africa (SEA), a consultative nongovernmental organisation promoting the development of a low carbon economy throughout Southern Africa by providing research, capacity building, and assistance with project implementation. Researchers at SEA had evaluated 134 electricity saving options on three criteria: electricity savings, capital cost, and the ease of making the change implied by the new technology or behaviour. There is substantial support for these attributes in previous literature around household energy use $[1,8,15]$, and these were confirmed in a workshop session with researchers at the ERC.

Measurable attributes had already been defined for each of the criteria. Electricity savings are measured in the number of kilowatt hours saved per month by the introduction of an electricity saving option. Capital cost is a monetary cost (measured in Rands) of implementation. Ease of change had originally been defined using a three-point subjective rating scale ("easy", "medium", "difficult") but during the assessment of uncertainty a feeling arose that greater differentiation was needed and this scale was expanded to a seven-point constructed scale. 
The 134 electricity saving options are divided into 9 categories: water heating; lighting; cooking; fridge and freezer; heating, ventilation and cooling; laundry and dishes; renewable energy; standby (essentially, using appliances when needed); and others. Because the current analysis was intended as a pilot study, a set of 8 of the cooking options were chosen as alternatives. These alternatives, which contain new technologies as well as changes to behaviour, are:

1. Buy a solar cooker.

2. Buy a gas stove/oven.

3. Buy a "hotbox" (used for cooking rice, stews, etc.)

4. Use a pressure cooker for food that takes a long time to cook.

5. Use a microwave for cooking, instead of an oven.

6. Ensure that the size of the pot matches the size of the stove plate.

7. Keep oven reflectors clean.

8. Boil only as much water as needed when using the kettle.

For each option, mean attribute evaluations were provided by SEA - based on prior research and literature, market research, and subjective assessment. These are shown in Figure 1. Uncertainty around the attribute evaluations is not formally assessed, although in some cases ranges of possible values have been recorded in supplementary notes to the spreadsheet.

\subsection{Uncertainty representation}

Uncertainty exists around the evaluation of each of the cost, electricity savings, and ease of use attributes. Expected electricity consumption and savings typically depend on the implementation of the alternative or have only been partially studied and remain the subject of discussion. Frequencies of usage are also subject to substantial variation. Uncertainty (perhaps more accurately labelled 'imprecision') exists around costs for some alternatives because these are described only in broad terms and do not specify the exact make or distributor, both of which will affect costs. A more precise definition of alternatives was not considered to be appropriate for the media campaign. Evaluations of ease of change are also subject to substantial variation.

A choice of uncertainty format was made based on a discussion involving the facilitator and ERC research group. Probability distributions were felt to be too time- and effortconsuming to construct, particularly bearing in mind that the application might ultimately be applied to a large number of alternatives. After a brief discussion, the group decided to assess uncertainty around the mean evaluations using the $5 \%$ and $95 \%$ quantiles for each attribute evaluation. This assessment was performed by one member of the group, in some cases assisted by ranges provided in an accompanying note to the mean evaluations (see Figure 1). The assessment of precise quantiles was felt to be difficult at the extremes, and after some discussion evaluations were self-reported as "not quite best" and "not quite worst" cases. The full value tree for the problem is shown in Figure 2 using a format which shows performance at different quantiles as higher-level criteria. The final evaluations are given in Table 1. 


\begin{tabular}{|c|c|c|c|c|c|c|c|c|c|}
\hline$\infty$ & $\begin{array}{l}\mathscr{\Xi} \\
\text { ळ }\end{array}$ & 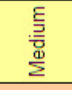 & 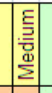 & 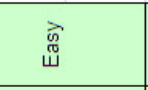 & $\begin{array}{l}\vec{J} \\
\text { ய }\end{array}$ & 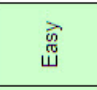 & 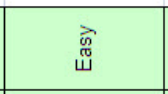 & \begin{tabular}{|l}
$\vec{\sigma}$ \\
யू
\end{tabular} & $\begin{array}{l}\vec{心} \\
\text { ய }\end{array}$ \\
\hline$\propto$ & 范 & 呈 & 㰻 & כे & 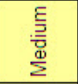 & 亏े & పे & పे & 亏े \\
\hline o & 造 & 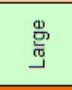 & : & 总 & 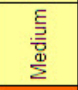 & $\begin{array}{l}\overline{\overline{0}} \\
\stackrel{\bar{E}}{\bar{D}} \\
\end{array}$ & $\begin{array}{l}\underline{\underline{\underline{\underline{z}}}} \\
\frac{\overline{\underline{\theta}}}{2}\end{array}$ & \begin{tabular}{|c|}
$\overline{\bar{\sigma}}$ \\
$\tilde{5}$ \\
$\bar{\omega}$
\end{tabular} & 离 \\
\hline ० & 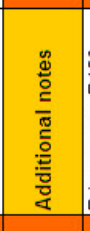 & 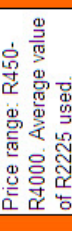 & & 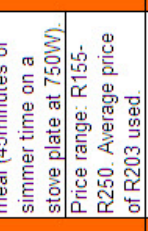 & & & 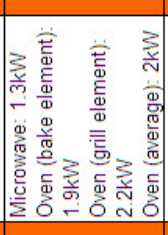 & & \\
\hline$\Sigma$ & 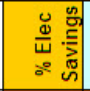 & $\stackrel{\circ}{\circ}$ & ㅇํㅇ & 今̊ & 号 & 亡ั & 品 & & ๕̊ \\
\hline$\checkmark$ & 空 & 苛 & जे & 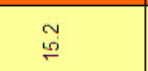 & జอ & 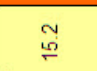 & 芦 & 吉 & 우 \\
\hline$\checkmark$ つ恋 & 올 & 量 & $\begin{array}{l}0 \\
\\
0\end{array}$ & 응 & $\begin{array}{l}8 \\
0 \\
0\end{array}$ & 品 & : & 8 & 홍 \\
\hline $\begin{array}{rl}- & 0 \\
-3 & 0\end{array}$ & క & $\stackrel{i}{i}$ & ì & $\stackrel{\circ}{\circ}$ & $\stackrel{\circ}{-}$ & $\stackrel{\circ}{\circ}$ & $\stackrel{i}{i}$ & i & $\stackrel{\text { i }}{4}$ \\
\hline 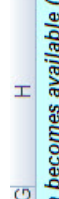 & 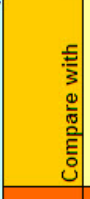 & 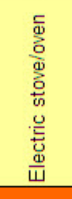 & 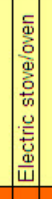 & 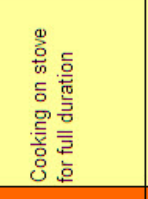 & 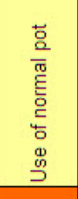 & 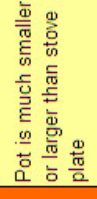 & 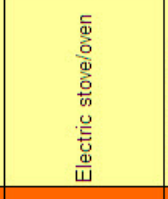 & 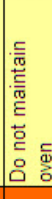 & 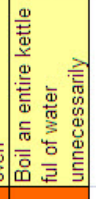 \\
\hline 4 & 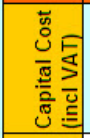 & 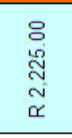 & 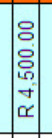 & $\begin{array}{l}\stackrel{\circ}{0} \\
\stackrel{\sim}{\tilde{N}} \\
\sim\end{array}$ & $\begin{array}{l}\stackrel{\circ}{0} \\
\stackrel{0}{0} \\
\stackrel{0}{\alpha}\end{array}$ & $\begin{array}{l}\stackrel{0}{0} \\
0 \\
\propto 0\end{array}$ & $\begin{array}{l}8 \\
0 \\
0\end{array}$ & $\begin{array}{l}\stackrel{8}{0} \\
0 \\
\square\end{array}$ & $\begin{array}{l}8 \\
0 \\
\propto \\
\propto\end{array}$ \\
\hline ш & 홀 & : & $:$ & $\overline{6}$ & $\stackrel{0}{i}$ & $\stackrel{+t}{\rightleftarrows}$ & $\stackrel{\infty}{\circ}$ & $\hat{\text { aे }}$ & $\hat{0}$ \\
\hline ○. & 黑垔 & $\begin{array}{l}8 \\
0 \\
0\end{array}$ & 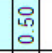 & స్ & $\stackrel{\text { L్ }}{0}$ & $\begin{array}{l}\infty \\
0 \\
0 \\
0\end{array}$ & 8 & $\begin{array}{c}8 \\
0 \\
0\end{array}$ & ¿ \\
\hline $0 \stackrel{0}{0}$ & 3्र & 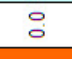 & $:$ & $\stackrel{\circ}{\circ}$ & $\stackrel{0}{-}$ & $\stackrel{\circ}{\circ}$ & $\stackrel{m}{-}$ & $\stackrel{\circ}{\mathrm{i}}$ & $\stackrel{i}{\mathrm{I}}$ \\
\hline 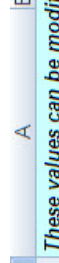 & 离 & 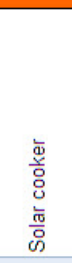 & 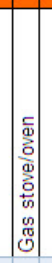 & 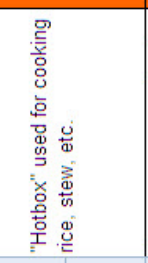 & 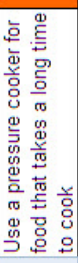 & 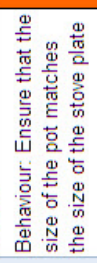 & 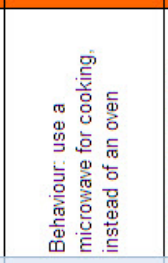 & 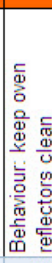 & 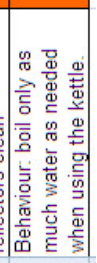 \\
\hline$\sqrt{2}$ & $\sim$ & & + & $\infty$ & $r$ & $\infty$ & $\sigma$ & 운 & $=:$ \\
\hline
\end{tabular}




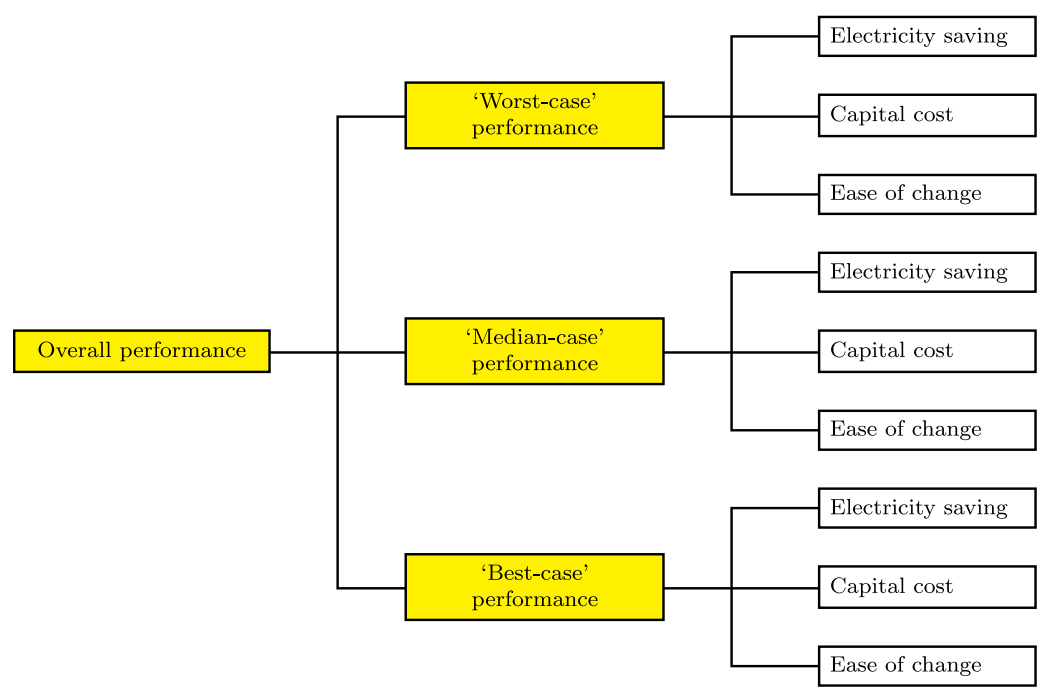

Figure 2: Value tree for selecting promising electricity savings options.

\begin{tabular}{|c|c|c|c|c|c|c|c|c|c|}
\hline \multirow[b]{2}{*}{ Alternative } & \multicolumn{3}{|c|}{ Elec savings } & \multicolumn{3}{|c|}{ Capital cost } & \multicolumn{3}{|c|}{ Ease of change } \\
\hline & Wor & Med & Best & Wor & Med & Best & Wor & Med & Best \\
\hline Solar cooker & 2 & 10 & 20 & 4500 & 2225 & 300 & 3 & 5 & 7 \\
\hline Gas stove/ovens & 30.4 & 30.4 & 30.4 & 20000 & 4500 & 1000 & 2 & 2 & 4 \\
\hline Hotbox & 2 & 9.1 & 25 & 250 & 200 & 150 & 2 & 3 & 4 \\
\hline Use pressure cooker & 5 & 7.6 & 12 & 1200 & 600 & 240 & 3 & 5 & 6 \\
\hline Pot matches plate & 1 & 3.8 & 5 & 0 & 0 & 0 & 1 & 2 & 3 \\
\hline Microwave & 8 & 10.6 & 13 & 875 & 250 & 150 & 4 & 5 & 7 \\
\hline Keep oven reflectors clean & 0.5 & 0.75 & 1 & 0 & 0 & 0 & 2 & 3 & 4 \\
\hline Boil water as needed & 4 & 11.3 & 14 & 0 & 0 & 0 & 2 & 3 & 4 \\
\hline
\end{tabular}

Table 1: Decision table for evaluation of electricity saving options

\section{$3 \quad$ Preference modelling}

The next phase of the analysis aims to use the attribute evaluations in Table 1 to assess the alternatives and select a shortlist that provide as many households as possible with at least one attractive option. In the absence of any information on household preferences, we base further modelling around inverse-preference models, in particular stochastic multi-criteria acceptability analysis (SMAA).

\subsection{A review of SMAA models}

The SMAA family of inverse-preference models are useful in applications such as this one where preference information is not precisely known. They provide information about the types of preferences (if any) that would lead to the selection of each alternative. That is, instead of asking 'which alternative is best given a particular set of preferences?', one asks 'what preferences might make a particular alternative the preferred one?'. A number of SMAA variants have been developed. These differ in terms of the preference model 
used and thus the type of preference information that is imprecisely known, but are all based upon Monte Carlo simulation from distributions which govern unknown preference parameters (and attribute evaluations). For example, SMAA variants are available for value function [25, 28], outranking [18], reference point [27, 11], and prospect theory [29] methods. The models described here are the value function based SMAA-2 [28], with or without an adjustment for the presence of ordinal criteria (SMAA-O, [26]).

Consider a decision problem consisting of $I$ alternatives $\left\{a_{1}, a_{2}, \ldots, a_{I}\right\}$ evaluated on $J$ attributes $\left\{c_{1}, c_{2}, \ldots, c_{J}\right\}$. Let $Z_{i j}$ be a random variable denoting the (possibly stochastic) attribute evaluation of $a_{i}$ on $c_{j}$, and $U$ be a multi-attribute utility function mapping the attribute evaluations of alternative $a_{i}$ (denoted $\boldsymbol{Z}_{i}$ ) to a real value using a weight vector $\boldsymbol{w}$. A joint density function $f_{X}(\boldsymbol{Z})$ governs the generation of the $Z_{i j}$ in the space $X \subseteq R^{I \times J}$, and a second joint density function $g(\boldsymbol{w})$ governs the generation of imprecise or unknown weights in the weight space $W$. Total lack of knowledge is usually represented by a uniform distribution in $W$.

Given a particular weight vector $\boldsymbol{w}$, the global utility of each alternative may be computed and a rank ordering of alternatives obtained. SMAA-2 is essentially based on simulating a large number ${ }^{2}$ of random weight vectors from $g(\boldsymbol{w})$ and observing the proportion and distinguishing features of weight vectors which result in each alternative obtaining a particular rank $r$ (usually the "best" rank, $r=1$ ). Let the set of weight vectors that result in alternative $a_{i}$ obtaining rank $r$ be denoted by $W_{i}^{r}$. SMAA-2 is based on an analysis of these sets of weights using a number of descriptive measures. Only the two utilised in this study are presented here:

Acceptability indices The rank- $r$ acceptability index $b_{i}^{r}$ measures the proportion of all simulation runs i.e. weight vectors, that make alternative $a_{i}$ obtain rank $r$.

Central weight vectors The central weight vector $\boldsymbol{w}_{i}^{c}$ is defined as the expected center of gravity of the favourable weight space $W_{i}^{1}$. It gives a concise description of the "typical" preferences supporting the selection of a particular alternative $a_{i}$, and in practice is computed from the empirical (element-wise) averages of all weight vectors supporting the selection of $a_{i}$ as the best alternative.

In applications where some of the criteria are measured on ordinal rather than cardinal scales, utilities are generated by randomly generating mappings between the ordinal and cardinal scales. At each iteration, utilities of 1 and 0 are assigned to the most and least favoured levels of the ordinal scale respectively, and $k-2$ randomly generated values from $U[0,1]$ are assigned (after being appropriately sorted) to the intermediate levels of the ordinal scale. This approach is known as SMAA-O [26].

\footnotetext{
${ }^{2}$ The exact number of Monte Carlo iterations that are required to achieve a given level is discussed in [38]. To estimate the acceptability index within $\xi$ of the true value with $95 \%$ confidence, one requires $1.96^{2} / 4 \xi^{2}$ iterations - so that 10000 iterations will usually be sufficient to achieve error bounds of $1 \%$. In the application reported in this paper computational time is not an issue and so 50000 iterations have been used.
} 


\subsection{Integrating simplified uncertainty formats with SMAA}

Adapting SMAA models to use simplified uncertainty formats is fairly straightforward. Each uncertain attribute is simply replaced by a number of lower-level attributes which capture the uncertainty in the evaluations on that attribute, using one of many possible simplified uncertainty formats - we discuss five formats: expected values; explicit risk attributes (e.g. variances or probability of performing below some specified cut-off); quantiles; fuzzy numbers; and scenarios (see [12] for more details). This transforms the decision problem into one having the same appearance as a deterministic decision problem, and may be treated by any of the existing SMAA models with some minor modifications. The precise form of these modifications depends on which uncertainty format (and hence simplified SMAA model) is being used. These are described in turn below, again using the value function based SMAA-2.

\subsubsection{Expected values}

Each uncertain evaluation $Z_{i j}$ is replaced by a single value, its expected value $E\left[Z_{i j}\right]$. The evaluation of $a_{i}$ is given by

$$
U_{i}^{(\mathrm{ev})}=\sum_{j=1}^{J} w_{j} u_{j}\left(E\left[Z_{i j}\right]\right)
$$

Examples using only expected values are [16] and [19], but it seems reasonable to suggest that a fair proportion of applications of multi-attribute value theory [22] would also fall into this category. In applying SMAA, based on expected values, attribute importance weights can be simulated as for SMAA-2. No random generation of attribute evaluations is required using this (or indeed in any other simplified SMAA) model.

\subsubsection{Explicit risk attributes}

Each uncertain evaluation $Z_{i j}$ is replaced by two values: its expected value $E\left[Z_{i j}\right]$ and an explicit risk measure $R_{i j}$ (for example, the variance of $Z_{i j}$ or the probability that $Z_{i j}$ does not exceed some specified target). The evaluation of $a_{i}$ is given by

$$
U_{i}^{(\text {risk })}=\sum_{j=1}^{J} w_{j} u_{j}\left(E\left[Z_{i j}\right]\right)-\sum_{j=1}^{J} w_{i j}^{R} R_{i j},
$$

where $R_{i j}$ is a measure of the 'risk' of $Z_{i j}$ and $w_{i j}^{R}$ is a 'risk weight' for $R_{i j}$. In this general formulation the risk weights may depend on alternatives as well as attributes. The use of variances is standard in (single-attribute) portfolio optimization [31]; multi-attribute applications are reported in $[10,23]$ and [2]. Probabilities of obtaining performance below a cut-off have also been used to measure risk $[5,9,36]$.

When applying SMAA using explicit risk attributes to represent uncertain attribute values, the simulation of weights can take several forms depending on the risk model chosen. If the variance weights proposed by Kirkwood [23] are used i.e. $w_{i j}^{R}=(-1 / 2) w_{j} u_{j}^{\prime \prime}\left(E\left[Z_{i j}\right]\right)$, this would require the same simulation of $J$ weights at each iteration as for SMAA-2. If other risk measures (like the probability of poor performance) are used, the most lenient 
approach - in terms of giving each alternative the maximum chance of obtaining one of the best ranks - is to randomly generate the risk weights $w_{j}^{R}$ and attribute importance weights $w_{j}$ together $i . e$. generate $2 J$ weights at each iteration. This would require that the $R_{i j}$ on each attribute be scaled beforehand to lie between 0 and 1 (assuming the utility functions $u_{j}$ do the same to the expected values). Any further weight restrictions (e.g. that risk weights should be some multiple of attribute importance weights) may be specified as required.

A potentially attractive approach is to use ordinal assessments of the risk of each attribute. This would require, for each uncertain attribute, only that the decision maker rank the set of alternatives from most to least risky. It may often be easier for a decision maker to make these ordinal assessments of uncertainty rather than to assess it quantitatively, particularly in the early stages of the decision process or where getting extensive participation from the decision maker is difficult.

\subsubsection{Quantiles}

In practical decision analysis it is common to represent probability distributions using three to five quantiles [13]; this is the basis for the well-known bisection and interval elicitation methods [35]. In a quantile-based model each uncertain evaluation $Z_{i j}$ is replaced by $N_{q}$ values, where $N_{q}$ is the number of quantiles used. The evaluation of $a_{i}$ is given by

$$
U_{i}^{\text {(quan) }}=\sum_{k=1}^{N_{q}} \sum_{j=1}^{J} w_{j k} u_{j k}\left(z_{i j}^{\left(q_{k}\right)}\right),
$$

where $q_{k}$ refers to a specific quantile, $z_{i j}^{\left(q_{k}\right)}$ is the $q_{k}$-th quantile of $Z_{i j}$, and $w_{j k}$ and $u_{j k}$ are respectively the weight and marginal utility function associated with quantile $q_{k}$ and attribute $c_{j}$.

When applying SMAA, quantiles $w_{j k}$ may be simulated directly or as $w_{q_{k}} w_{j \mid q_{k}}$, where $w_{j \mid q_{k}}$ is a relative criterion weight [4] denoting the weight of criterion $c_{j}$ at quantile $q_{k}$, with $\sum_{j} w_{j \mid q_{k}}=1$. Other weight restrictions (e.g. equal quantile weights) may be specified as required as part of the simulation. One restriction which may be particularly useful is to restrict quantile weights to be those identified by Keefer and Bodily in [21] i.e. to use quantile weights $w_{0.05}=w_{0.95}=0.185, w_{0.5}=0.63$, and then generate only the relative criterion weights $w_{j \mid q_{k}}$.

\subsubsection{Fuzzy numbers}

Fuzzy set theory is a general theory for the modelling of imprecision. The fundamental notion in fuzzy set theory is that imprecision manifests itself as an arbitrariness in establishing precise boundaries for a set of interest, allowing set membership to be considered a matter of degree. There are a large number of fuzzy value function approaches [7], but in general each uncertain evaluation $Z_{i j}$ is replaced by a fuzzy number, usually triangular 
or trapezoidal. The fuzzy global evaluation of $a_{i}$ is given by

$$
\tilde{U}_{i}=\left[\sum_{j=1}^{J} w_{j} u_{j}\left(z_{i j}^{\left(q_{1}\right)}\right), \sum_{j=1}^{J} w_{j} u_{j}\left(z_{i j}^{\left(q_{2}\right)}\right), \sum_{j=1}^{J} w_{j} u_{j}\left(z_{i j}^{\left(q_{3}\right)}\right), \sum_{j=1}^{J} w_{j} u_{j}\left(z_{i j}^{\left(q_{4}\right)}\right)\right]
$$

for trapezoidal fuzzy numbers; for triangular fuzzy numbers $z_{i j}^{\left(q_{2}\right)}=z_{i j}^{\left(q_{3}\right)}$. These fuzzy evaluations can be ranked using a number of methods [7]. In applying SMAA with fuzzy attribute values, a set of $J$ attribute importance weights must be generated at each iteration, as for the conventional SMAA-2. The chosen method for ranking the fuzzy global evaluations may also have weighting parameters that may be randomly generated if necessary. These would presumably be simulated independently of the attribute importance weights.

\subsubsection{Scenarios}

Uncertain outcomes may also be represented using a set of scenarios - incomplete but internally-consistent narratives of how the future might unfold. The use of 'scenario planning' [40, 39] emphasises gaining insight into the problem and generating novel actions. Multi-attribute scenario models [14, Ch. 14] apply a deterministic multi-attribute model within each scenario, followed (possibly) by an aggregation over scenarios [37]. Applications are reported in $[30,17,32]$. In a scenario-based model each uncertain evaluation $Z_{i j}$ is replaced by $N_{s}$ values, where $N_{s}$ is the number of scenarios used. The evaluation of $a_{i}$ is given by

$$
U_{i}^{(\mathrm{scen})}=\sum_{k=1}^{N_{s}} \sum_{j=1}^{J} w_{j k} u_{j k}\left(z_{i j}^{\left(s_{k}\right)}\right)
$$

where $s_{k}$ refers to a specific scenario, $z_{i j}^{\left(s_{k}\right)}$ is the evaluation of alternative $a_{i}$ on attribute $c_{j}$ in scenario $s_{k}$, and $w_{j k}$ and $u_{j k}$ are respectively the weight and marginal utility function associated with attribute $c_{j}$ under the assumption of scenario $s_{k}$. As for quantiles, the weights $w_{j k}$ may be simulated directly or as $w_{s_{k}} w_{j \mid s_{k}}$, although it must be noted that the practical interpretation and assessment of scenario weights has not been fully resolved (see [37] for details). Other weight restrictions (e.g. equal scenario weights) may be specified as required.

\section{Results}

Results were obtained by applying a SMAA-O model to accommodate the ordinal ease of change attribute. Utility functions for the two cardinal attributes were randomly generated as per the SMAA-2 model to be convex below a reference point and concave above it, according to general prospect theory principles [20]. Costs were defined as negative profits so that utility functions are increasing in all three attributes. Reference levels reflect the status quo of no electricity savings and zero cost, so that utility functions for the electricity savings attribute(s) are generated to be between linear and moderately concave; and utility functions for the capital cost attribute(s) are generated to be between linear and moderately convex. Utility functions for the ordinal ease of change attribute are 
simulated by randomly generating sets of cardinal values that are consistent with the known ordinal values as per SMAA-O. Three models were used here to show the impact of uncertainty representation and quantile weight generation, although typically (i.e. outside of the current 'action research' context) not all of these would be shown to the decision maker.

Model 1 uses the mean/median evaluations only, as described by Section 3.2.1 and equation (1).

Model 2 uses the full set of evaluations in Table 1 with Keefer-Bodily quantile weights, as described by Section 3.2.3 and equation (3) with $w_{0.05}=w_{0.95}=0.185$ and $w_{0.5}=0.63$.

Model 3 uses the full set of evaluations in Table 1 with quantile weights allowed to vary freely, also as described by Section 3.2.3 and equation (3) with values for $w_{j k}$ generated randomly as part of the SMAA simulations.

Figure 3 displays the rank acceptability indices for each alternative obtained from the three models. These show the share of the different preferences i.e. weights, that support an alternative for a particular rank 1 (best) through 8 (worst). Note that results for Model 1 are equivalent to those of Model 3 (medians only) shown in Figure 3(d).

The most promising alternatives are those with larger acceptability indices for the best ranks, which appear towards the bottom-right corner of each of the plots. There was reasonably strong agreement between the models on the most promising alternatives. All models identify gas stoves/ovens, matching pots to stove plate sizes, and boiling water only as needed as alternatives that potentially appear first in a preference order. The sum of the rank-one acceptability indices for these three alternatives is (in Model 3) 0.91, signifying that $91 \%$ of household preferences lead to the selection of one of these alternatives. Hotboxes and microwaves also show substantial acceptability indices for second and third ranks, with hotboxes in particular becoming relatively more favourable in the models using quantiles (Models 2 and 3). The other three alternatives - keeping oven reflectors clean, pressure cookers, and solar cookers - tend to occupy lower ranks. The main difference between the results of Model 2 and Model 3 is that the two alternatives with the highest rank-one acceptabilities - gas stoves/ovens and boiling water as needed - swap ranks. Because the aim of the analysis is to identify a shortlist of promising alternatives the reversal was not considered to be particularly important, but is caused by boiling water being favoured by a greater range of quantile weights, particularly those placing more weight on the 'best case' quantile than the Keefer-Bodily weights do.

In an informal feedback discussion, the group indicated that they found the acceptability results easy to understand and interpret. They identified the ability of the SMAA model to select a diverse set of promising alternatives with minimal additional information as its main benefit, and it became fairly clear during the analysis that a promising shortlist of alternatives should include gas stoves/ovens, matching pots to stove plate sizes, boiling water only as needed, and hotboxes. The selection of promising alternatives was experienced as fairly easy and was based on (a) the acceptability indices for the best ranks and (b) the robustness of the rank of an alternative to changes in preferences. The latter criterion was 


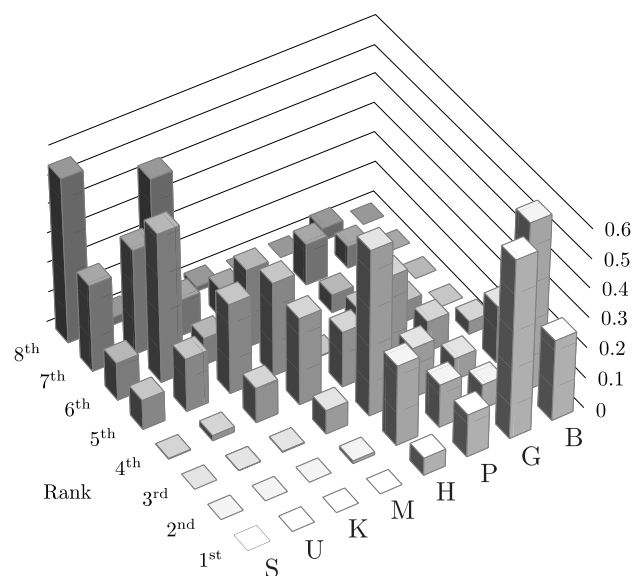

(a) Model 2

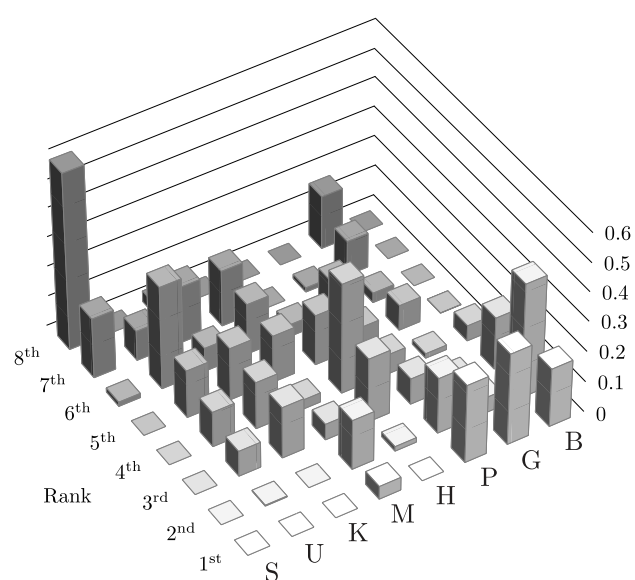

(c) Model 3, worst cases only

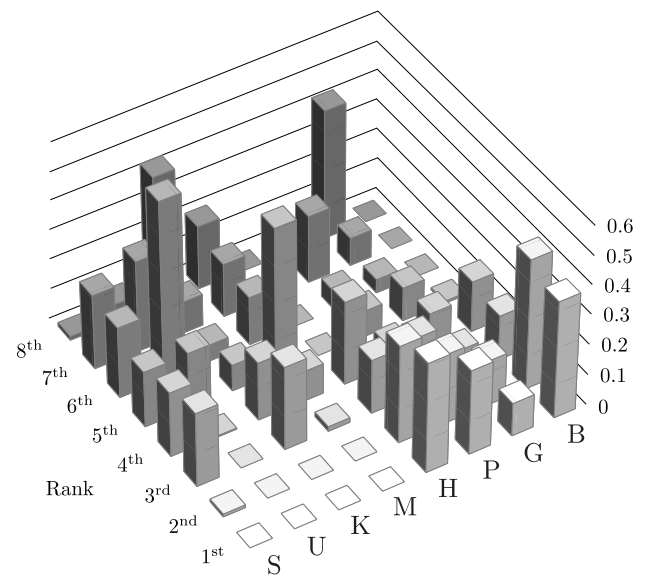

(e) Model 3, best cases only

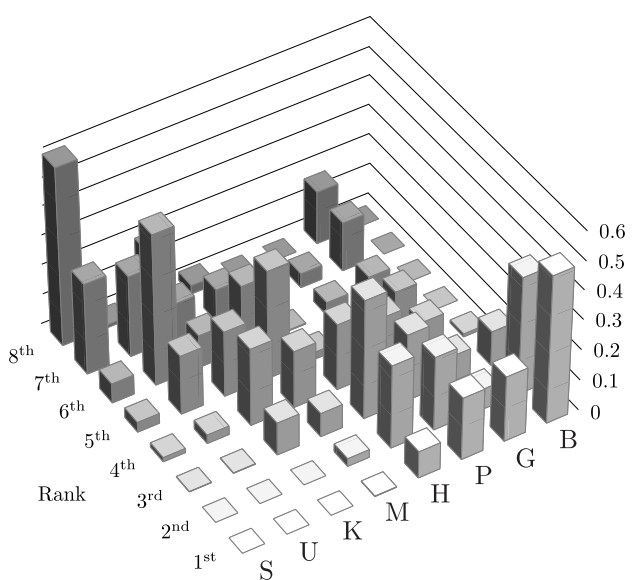

(b) Model 3

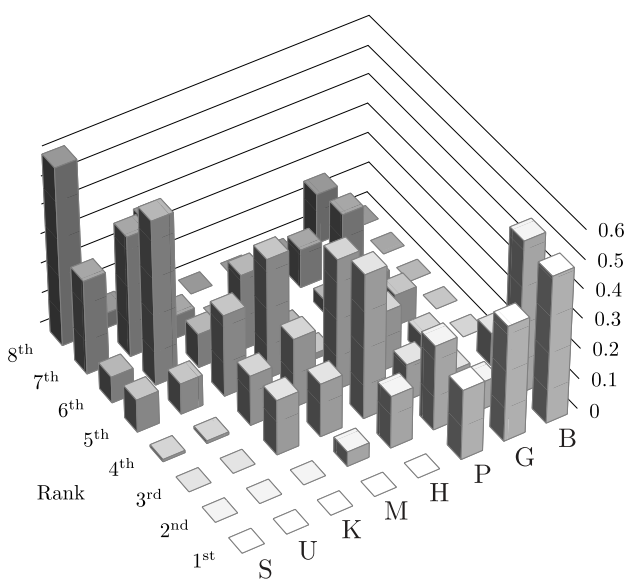

(d) Model 3, median cases only
Key:

\begin{tabular}{r|l} 
S & Solar cooker \\
U & Use pressure cooker \\
K & Keep stove clean \\
M & Microwave \\
H & Hotbox \\
P & Pot matches plate \\
G & Gas stove/oven \\
B & Boil water as needed
\end{tabular}

Figure 3: Acceptability indices for electricity savings options obtained with an adapted SMAA$O$ model. 
felt to be of importance given the aim of promoting alternatives that would be viewed as acceptable by as many of the target audience as possible. Hotboxes and boiling water as needed were identified as the most robust of the promising options, and gas stoves/ovens and matching pot and plate size as relatively sensitive to preferences. The poor robustness of microwave ovens also played an important role in the group's decision to exclude this alternative from the shortlist. The extra (within-quantile) acceptability information provided by Model 3 was considered useful in better understanding the performance of the alternatives but in this instance did not affect the selection of alternatives.

Figure 4 displays the central weights for each alternative with a non-zero rank-one acceptability index - these show the typical (average) weights that make each alternative most preferred. A large shaded area for an attribute indicates that a greater weight is allocated to that attribute in the central weight vector. All figures are based upon Model 3. Very similar attribute importance weights were obtained using Model 2, and these are thus not shown here.

The central weight vectors shown in Figure 4 provide insight into the reasons why certain alternatives may be preferred. Figure 4(a) displays the central weights for the three attributes, and shows that:

- The use of a hotbox tends to be preferred by decision makers who place roughly equal weights on the three attributes, with slightly more weight being placed on electricity savings.

- Matching pots to plate sizes tends to be preferred by decision makers with relatively large weights on ease of change, and small weights on electricity savings.

- The use of a microwave tends to be preferred by decision makers with relatively large weights on electricity savings, and small weights on ease of change.

- Boiling water only as needed tends to be the preferred action of decision makers who place roughly equal weights on the three attributes (as for hotboxes), but with slightly more weight being placed on cost.

- The use of gas stoves/ovens tends to be the preferred action of decision makers with relatively small weights on cost and larger weights on electricity savings.

The central attribute weights were easily understood and interpreted by the group, and confirmed "common sense" descriptions of the alternatives. Note that Figure 4(d) shows that if only medians are used (Model 1), then no descriptions are provided for hotboxes or microwaves because their rank-one acceptabilities are in that case zero. In this application the addition of other quantiles therefore had a substantive impact on the information that could be displayed. The weights in Figure 4(a) can also be thought of as describing different decision maker "typologies." These typologies might be used to customise intervention recommendations. This possibility was identified as a second benefit of the analysis.

Figure 4(b) shows the central quantile weights for Model 3. The approximately equal quantile weights obtained for matching pot and stove plate size, boiling water as needed, and gas stoves/ovens, together with these alternatives' large rank-one acceptability indices, suggest wide support (rather than quantile weights having to be equal). The use of a hotbox tends to be preferred if more weight is placed on the 'best-case' evaluations, and the use of a microwave tends to be preferred if more weight is placed on the 'worst-case' 


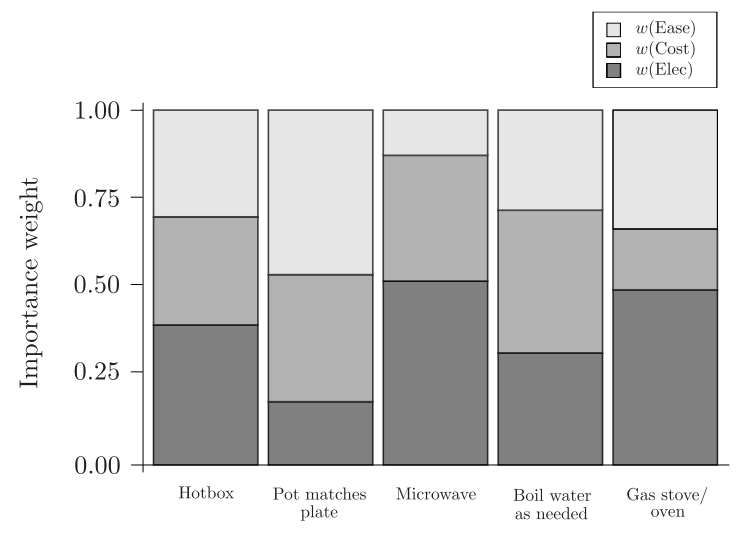

(a) Attribute weights (all quantiles)

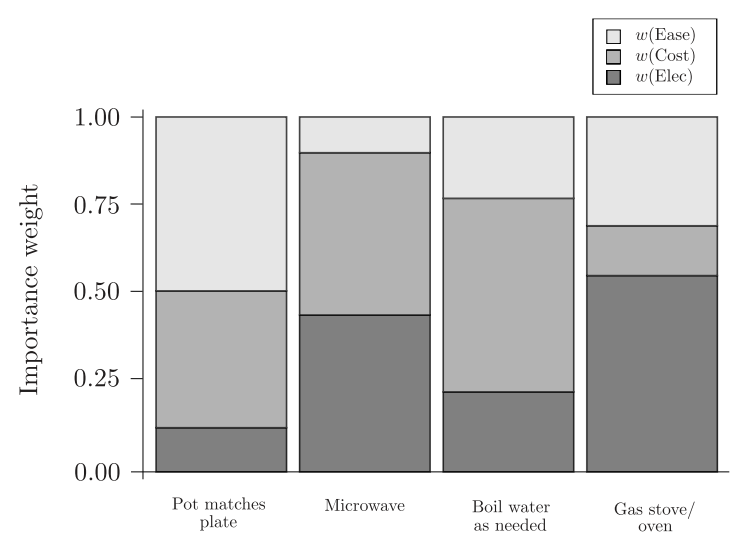

(c) Worst cases only

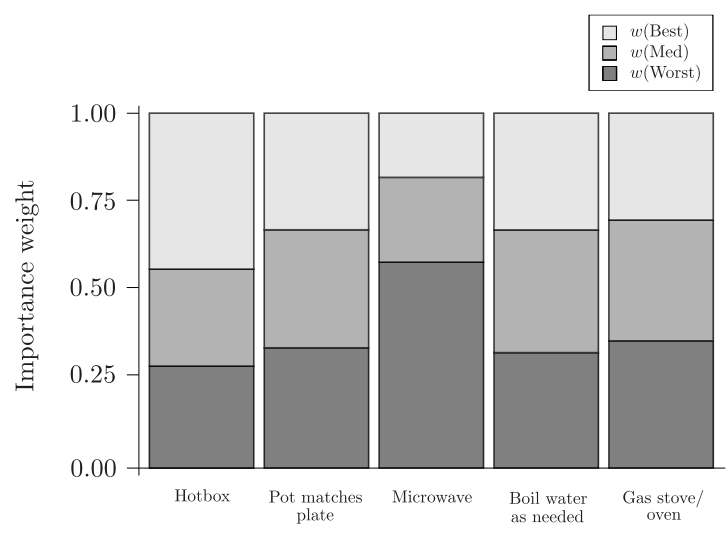

(b) Quantile weights (all attributes)

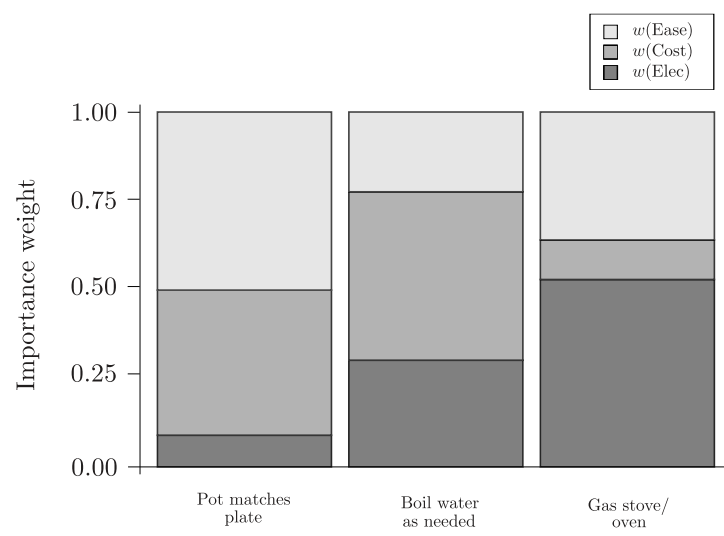

(d) Median cases only
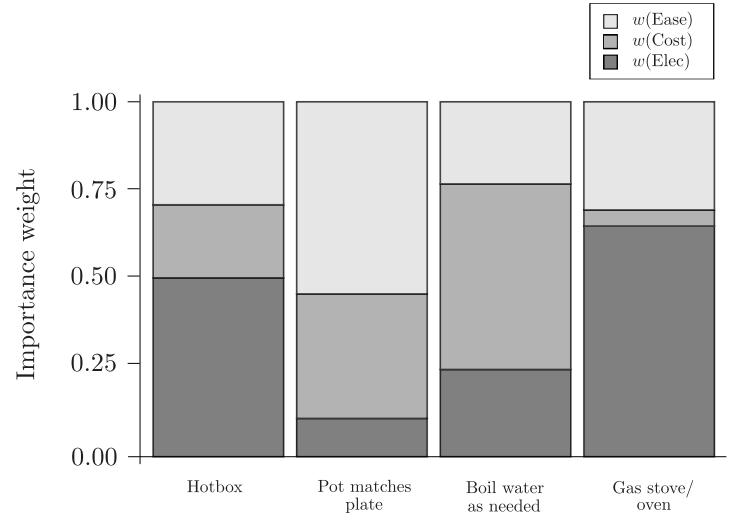

(e) Best cases only

Figure 4: Central weight vectors for electricity savings options obtained with an adapted SMAA-O model. All figures are based upon Model 3. 
evaluations.

The information provided by the central quantile weights was considered of relatively minor importance when compared with that provided by the central attribute weights, although knowing that the attractiveness of a hotbox depends quite heavily on being applied 'ideally' was felt to be a practically useful insight. It was noted that Model 2 (with constant KeeferBodily weights) provided essentially the same conclusions, although the group accepted that results would not necessarily be so similar in other cases. Finally, we note that initially a presentation of joint attribute and quantile weights $w_{j k}$ was attempted (not shown here). Interpretation of these weights was experienced as difficult and the group preferred the aggregated results shown in Figure 4(a) and (b).

The ERC project is in its early stages but the group is satisfied that the SMAA approach can contribute to the design of targeted media around electricity savings options, by supporting the decision of which options to include in the media. Similar analyses to the one performed here for cooking interventions are to be carried out for other categories of energy e.g. cooking, lighting, heating) in the near future. The choice of media platform(s) and the design of the media should then be scheduled. In the longer term the project aims to evaluate the impact of targeted campaigns on awareness of energy-related issues and making consumers actively involved in decision making around their own energy-related behaviour, but it is not yet possible to assess whether the shortlists of alternatives provided by the SMAA model are effective in helping to achieve these goals.

\section{Conclusions}

It was clearly possible to employ a simplified SMAA model in a practical decision problem. In our application, the inclusion of quantiles around the original estimates of expected values was felt to add value to the analysis. Two alternatives that were dominated at their mean values (and hence had no central weight vectors) became relatively more attractive once quantiles were included. This provided a richer set of electricity user "typologies". The group also felt it was useful to be able to assess the extent to which the attractiveness of options depended on the manner in which they are applied. Media for options like the hotbox which are more attractive at the best-case quantile should be accompanied by greater educational material than those options which give relatively similar performance over quantiles, for example.

In terms of difficulties, the assessment of quantiles did take some time and was experienced as moderately difficult although also useful as a learning process. Although 5\% and 95\% quantiles were requested, the researcher preferred to think of evaluations as "almost" worst- and best-case performances respectively. The interpretation of quantile weights required some care, and the joint interpretation of attribute and quantile weights i.e. of the $w_{j k}$, was experienced as overly complex. Instead, decision makers found it easier to interpret attribute weights and quantile weights separately. Although no elicitation of weight information was performed, the same difficulties and responses might be expected to apply there too.

SMAA models predominantly address internal uncertainty, particularly uncertainty relat- 
ing to the preferences of the decision maker [37]. It may be that many of the conditions that suggest the use of the SMAA approach for resolving internal uncertainty might also suggest the use of a simplified format for resolving external uncertainty. The application reported in this paper, for example, was characterised by constraints on the time and effort that stakeholders were willing to devote to the decision process, and by a lack of knowledge about the exact effects that various technologies and behavioural interventions might have on a household's electricity use. In such cases the simplified SMAA models developed in Section 3.2 may be useful additions to the decision support toolbox in general and to the family of SMAA models in particular.

\section{References}

[1] Abrahamse W, Steg L, Vlek C \& Rothengatter T, 2005, A review of intervention studies aimed at household energy conservation, Journal of Environmental Psychology, 25(3), pp. 273-291.

[2] Ballestero E, 2001, Stochastic goal programming: A mean-variance approach, European Journal of Operational Research, 131(3), pp. 476-481.

[3] Bana e Costa C, 1986, A multicriteria decision aid methodology to deal with conflicting situations on the weights, European Journal of Operational Research, 26(1), pp. 22-34.

[4] Belton V \& Stewart T, 2002, Multiple criteria decision analysis: An integrated apporach, Kluwer Academic Publishers, Boston (MA).

[5] Bordley C \& Kirkwood C, 2004, Multiattribute preference analysis with performance targets, Operations Research, 52(6), pp. 823-835.

[6] Bryman A \& Bell E, 2002, Business research methods, Oxford University Press, Bath.

[7] Chen SJ \& Hwang CL, 1992, Fuzzy multiple attribute decision making: Methods and applications, Springer-Verlag, Berlin.

[8] Davis S \& Durbach I, 2010, Modelling household responses to energy efficiency interventions via system dynamics and survey data, ORiON, 26(2), pp. 79-96.

[9] De P, Acharya D \& SAHU K, 1982, A chance-constrained goal programming model for capital budgeting, Journal of the Operational Research Society, 33(7), pp. 635-638.

[10] Donckels R, 1977, Regional multiobjective planning under uncertainty: A stochastic goal programming formulation, Journal of Regional Science, 17(2), pp. 207-216.

[11] Durbach I, 2006, A simulation-based test of stochastic multicriteria acceptability analysis using achievement functions, European Journal of Operational Research, 170(3), pp. 923-934.

[12] Durbach I \& Stewart T, 2012, Modeling uncertainty in multi-criteria decision analysis, European Journal of Operational Research, 223(1), pp. 1-14.

[13] Garthwaite P, Kadane J \& O'Hagan A, 2006, Statistical methods for eliciting probability distributions, Journal of the American Statistical Association, 100(6), pp. 680-701.

[14] Goodwin P \& Wright G, 1998, Decision Analysis for Management Judgement, $2^{\text {nd }}$ Edition, John Wiley \& Sons, Chichester.

[15] Gyberg P \& Palm J, 2009, Influencing households' energy behaviour - How is this done and on what premises?, Energy Policy, 37(7), pp. 2807-2813. 
[16] Hajkowicz S \& Higgins A, 2008, A comparison of multiple criteria analysis techniques for water resource management, European Journal of Operational Research, 184(1), pp. 255-265.

[17] Haralambopoulos D \& Polatidis H, 2003, Renewable energy projects: Structuring a multi-criteria group decision-making framework, Renewable energy, 28(6), pp. 961-973.

[18] Hokkanen J, Lahdelma R, Miettinen K \& Salminen P, 1998, Determining the implementation order of a general plan by using a multicriteria method, Journal of Multi-Criteria Decision Analysis, 7(5), pp. 273-284.

[19] Janssen R, 2001, On the use of multi-criteria analysis in environmental impact assessment in the Netherlands, Journal of Multi-Criteria Decision Analysis, 10(2), pp. 101-109.

[20] Kahneman D \& Tversky A, 1979, Prospect theory: An analysis of decision under risk, Econometrica, 47(2), pp. 263-291.

[21] Keefer D \& Bodily S, 1983, Three-point approximations for continuous random variables, Management Science, 29(5), pp. 595-609.

[22] Keeney R \& Raiffa H, 1993, Decisions with multiple objectives: Preferences and value tradeoffs, Cambridge University Press, Cambridge.

[23] KIRKWOOD C, 1992, Estimating the impact of uncertainty on deterministic multiattribute evaluation, Management Science, 38(6), pp. 819-826.

[24] Kotler P \& Andreasen A, 1996, Strategic marketing for nonprofit organizations, Prentice-Hall, New Jersey (NJ).

[25] Lahdelma R, Hokkanen J \& Salminen P, 1998, SMAA - Stochastic multiobjective acceptability analysis, European Journal of Operational Research, 106(1), pp. 137-143.

[26] Lahdelma R, Miettinen K \& Salminen P, 2003, Ordinal criteria in stochastic multi-criteria acceptability analysis (SMAA), European Journal of Operational Research, 147(1), pp. 117-127.

[27] Lahdelma R, Miettinen K \& Salminen P, 2005, Reference point approach for multiple decision makers, European Journal of Operational Research, 164(3), pp. 785-791.

[28] Lahdelma R \& Salminen P, 2001, SMAA-2: stochastic multi-criteria acceptability analysis for group decision making, Operations Research, 49(3), pp. 444-454.

[29] Lahdelma R \& Salminen P, 2009, Prospect theory and stochastic multicriteria acceptability analysis (SMAA), Omega, 37(5), pp. 961-971.

[30] Lootsma F, Boonekamp P, Cooke R \& Van Oostvoonn F, 1990, Choice of a long-term strategy for the national electricity supply via scenario analysis and multi-criteria analysis, European Journal of Operational Research, 48(2), pp. 189-203.

[31] Markowitz H, 1952, Portfolio selection, Journal of Finance, 7(1), pp. 77-91.

[32] Montibeller G, Gummer H \& Tumidei D, 2006, Combining scenario planning and multi- criteria decision analysis in practice, Journal of Multi-criteria Decision Analysis, 14(1), pp. 5-20.

[33] Smil V, 2003, Energy at the crossroads: Global perspectives and uncertainties, MIT Press, Cambridge (MA).

[34] Solomon S, Qin D, Manning M, Chen Z, Marquis M, Averyt K, Tignor M \& Miller H, 2007, Contribution of working group I to the fourth assessment report of the intergovernmental panel on climate change, Cambridge University Press, Cambridge.

[35] Spetzler CS \& Stä̈l Von Holstein CAS, 1975, Probability encoding in decision analysis, Management Science, 22(3), pp. 340-358. 
[36] Stewart T, 1998, Measurements of risk in fisheries management, ORiON, 14(1/2), pp. 1-15.

[37] Stewart T, 2005, Dealing with uncertainties in MCDA, pp. 445-470 in Figueira J, Greco S \& Ehrgott M (EDS), Multiple Criteria Decision Analysis: State of the Art Surveys, Springer, Boston (MA).

[38] Tervonen T \& Lahdelma R, 2007, Implementing stochastic multicriteria acceptability analysis, European Journal of Operational Research, 178(3), pp. 500-513.

[39] VAn Der HeiJden K, 1996, Scenarios: The art of strategic conversation, John Wiley \& Sons, New York (NY).

[40] Wack P, 1985, Scenarios: Uncharted waters ahead, Harvard Business Review, September/October, pp. 73-89. 